\title{
A Brief Introduction to the Control System of a Ship’s Propulsion Device
}

\author{
You Xiaoguang, Cao Zhaofei, Tao Fei \\ China Satellite Maritime Tracking and Control Department, Jiangyin, 214431, China
}

Keywords: Main propulsion device; control system; pitch

\begin{abstract}
In this paper, the control system of a ship owner propulsion device is introduced. The main control cabinet, main control system, gear box and shaft monitoring board are described in detail, and the basic control principle of pitch is clarified. The emergency control method of pitch is given, which is convenient for the relevant personnel to systematically understand the whole control system and provide reference for similar system learning.
\end{abstract}

\section{Introduction}

The ship propulsion device is a device that propels the ship's voyage, which is powered by the ship's main body, transmitted to the propeller through the transmission mechanism and shafting, and the thrust of the propeller in the water is transmitted to the hull to propel the ship. The main propulsion of the ship generally includes the main engine, transmission mechanism, shaft and propeller.

\section{Overview of the main propulsion control system}

The main propulsion control system is an important part of the ship to realize the automatic control of the main propulsion diesel engine, gear box, propeller and shaft attachment. Its main functions include data acquisition, system joint control and system main condition monitoring.

Figure 1 shows the AT2000 main propulsion control system diagram, the system mainly by the bridge operating unit (including the operation panel, operating handle and dashboard), the console operating unit, the main control cabinet, host monitoring system SoCoSone, gearbox and Shaft monitoring board and other components.

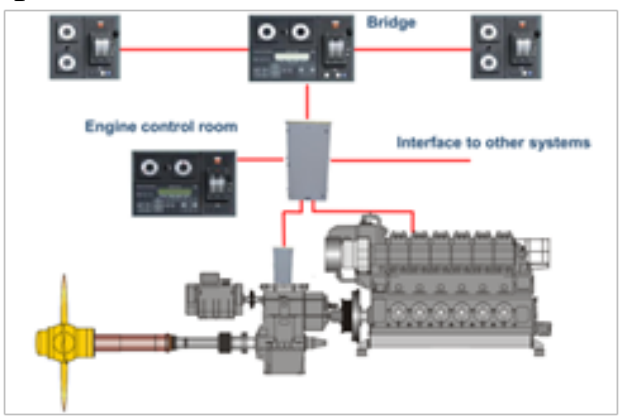

Figure 1. Main propulsion system control system diagram

The operation panel is mainly used for the operation of the cab and the control room in the control room, and the backup control operation, the dashboard and the operation panel will have the relevant data display. When the system is faulty, the system's on-site emergency control can be implemented on the gearbox and adjustable oar integrated operation panel (OP-GP). It is important to note that the operation of the system is based on the completion of the main propulsion engine has been completed, that is, the system is not responsible for the start of the main diesel engine operation, its stop operation is also limited to emergency shutdown, the main safety of diesel Managed by the host monitoring and security system SaCoSone.

The power supply of the whole system is from 220VAC under the centralized control station, and 
then the $220 \mathrm{~V}$ AC power is rectified to $24 \mathrm{~V}$ DC for two-way system for the uninterruptible power supply unit. The unit with backup battery can be in the process of electricity for the system to provide uninterrupted power supply. The details of each of the cables, such as W003, are available in the wiring diagram of the main propulsion control system.

The main control cabinet of the main propulsion system is the core of the entire control system and is also located in the centralized control room. As shown below is the AT2000 main propulsion control system main control cabinet layout.

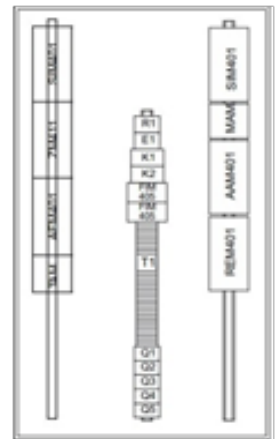

Figure 2. The main control cabinet layout

The controller cabinet includes a microprocessor module (ZM411), a dual channel serial interface module (SIM401), a speed adaptation module (TAM), an 8-channel analog input module (AEM401), input/output for digital input/output Adapter module (MAM401), 4-channel analog output module (AAM401), 16-channel digital input and 8 relay output module (REM401), 24V DC power supply filter module (FIM405) and so on. Q1 Q5 are provided by the 24V DC power supply after rectification, Q1 Q3 for the security system power supply, for the machine next to the detection, control and security system, Q4 Q5 for the propulsion system and promote the backup control system.

\section{Overview of Host Control Systems}

The SaCoSone system for the operation, alarm and control of the host is equipped with a monitoring and safety system. SaCoSone is composed of Sa: Safety; Co: Control; S: System; one: On Engine, a part of the English word composed of a compound word, meaning diesel engine safety and control system. It includes the following functions: host security system; host control system; host monitoring and alarm; host speed control system; solenoid valve drive; auxiliary control.

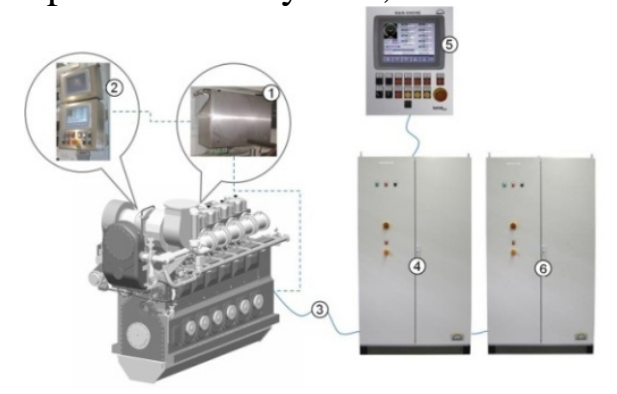

Figure 3. SaCoSone control system composition diagram

Figure 3 shows the physical composition of the SaCoSone control system, where:

(1). CU control unit

All sensors and associated operating equipment are connected to a control unit mounted on a diesel engine. It consists of two modules: one for safety control and one for diesel control, speed control and alarm. The two modules work independently and collect the relevant data of the diesel engine through a separate sensor.

(2). Local operator panel and remote control panel

Host installed next to the local operation screen, and equipped with anti-vibration pad, composed of two LCD screen, one with a prepared. The remote control panel is installed in the control room and has the same function as the local operator panel. 


\section{(3). System bus}

The SaCoSone system bus connects all modules of the system. The system bus is used for data exchange between modules, and data bus connections are made on the gateway module. The gateway module is connected to the input and output channels of the whole ship monitoring alarm, including the remote control panel and remote data transmission.

(4). Interface cabinet and auxiliary cabinet

The interface cabinet and the auxiliary cabinet provide the interface between the main control system and the main propulsion control system and other peripheral systems. It is also the power distribution unit of the main switchboard on board to the host control system.

\section{Gearbox and shaft monitoring board}

The servo valve is located in the gear box, and the servo piston is located inside the hub, so the gear box and shaft monitoring is concentrated together. The hardware is mainly composed of two, one is located on the gear box on the monitoring unit (ACM-GP) and screw closed loop servo unit ASP12, the other is located in the gear box near the gear box and adjustable oar integrated operation panel (OP- GP).

\subsection{Gear box monitoring unit and closed loop servo unit}

Figure 4 is located in the gear box on the monitoring unit and closed loop servo unit diagram. The internal arrangement of the monitoring unit cover is the same. Among them, ACM-GP is the monitoring unit board, which is used to collect the gear box; propeller and closed-loop servo unit ASP12 related parameters. J01-J31 for the terminal, the specific wiring can refer to the gear box instructions related content. All measurement data can be sent from the ACM-GP to the whole ship alarm system, when the test point exceeds the alarm value can be issued to the ship as needed alarm.

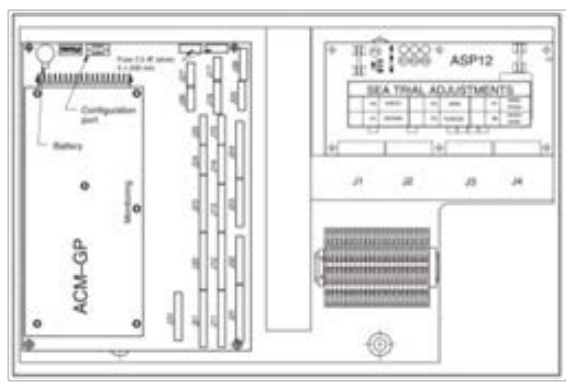

Figure 4. Gearbox on the monitoring unit and closed loop servo unit diagram

Closed loop servo unit ASP12 is used to realize the control of propeller pitch. There are three different control modes: remote control pitch control, standby control and machine control. In the remote control pitch control mode, the $4 \sim 20 \mathrm{~mA}$ set value command signal is received from the remote control system; in the standby control mode, the set value command signal is received from a single potentiometer in the main cab control handle. In the machine side control mode, the solenoid valve is directly controlled by the forward and reverse command switches on the machine side control panel, and the displacement sensor is used to indicate the actual propeller pitch. Closed-loop servo unit ASP12 can be achieved on the relevant closed-loop parameter adjustment.

\subsection{Gearbox and adjustable oar integrated operation panel}

The gearbox and adjustable oar integrated operator panel (OP-GP) have two functions, the upper part is used for on-site monitoring and the lower half is used for local control.

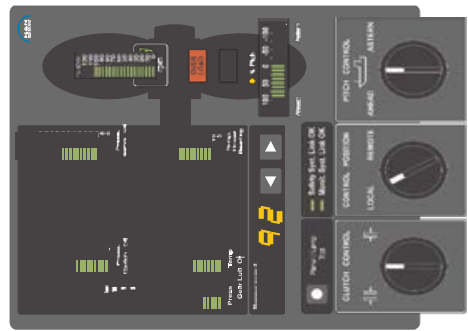

Figure 5. Gearbox and adjustable oar integrated operation panel 
The local monitoring section displays the measurement data of the gearbox and the propeller using a histogram. For easy overview, the histogram is designed to simulate the wireframe, showing the approximate position of the sensor. On the analog board, there will be a yellow lamp post showing the measured value. In addition to the display on the histogram, you can press the key to display the specific measurement data.

When the indicators labeled "SafetySyst.Link OK" and "Monit.Syst.LinkOK" are green on the operation panel, the communication with the ACS and ACM-GP is normal. If any of the Alpha common systems communicate with each other, the corresponding indicator will be extinguished. The specific measurement data on the panel comes from the sensor on the ACM-GP. When the sensor is faulty, the corresponding indicator bar on the integrated panel flashes, indicating that the corresponding sensor is faulty or the yellow LED lights that display the specific measurement data. When the fuel rack exceeds $100 \%$, the ACS module triggers 'OVER LOAD'. If the pitch is in place at this time, the operator must reduce the pitch to reduce the load on the diesel engine.

\subsection{Emergency control of propeller pitch}

When the closed loop servo control device cannot control the position of the propeller pitch, it will send the remote control system fault signal to the ship alarm system. At this time, the engine operator should switch the propeller pitch to the local control manual operation.

With the experience of the actual operation, when the host has reached the standby state, the propulsion device can be manually operated according to the following steps:

A). Start the diesel engine from the side panel of the diesel engine;

B). Manual operation of the diesel engine to increase the speed of the diesel engine, the speed set the value of the appropriate high, you can directly speed up to 750rpm;

C). Control the diesel engine and gearbox / propeller control (REMOTE) to local (LOCAL) control on the control panel of the gearbox and propeller;

D). Control the clutch row on the control panel of the gearbox and the propeller;

E). The actual pitch value is displayed on the in-place operation panel according to the cab command, the camera side command and the pitch relationship table, and the AHEAD and ASTERN command switches are operated on the local operation panel to adjust the pitch to the required value.

\section{Summary}

The safe and reliable operation of the main propulsion device is inseparable from the reliable control of the control system. The overall understanding of the main propulsion control system will help the engine manager understand and become familiar with the working principle of the main propulsion device and lay a good foundation for eliminating some simple faults. In this paper, the introduction of a ship owner propulsion control system can provide reference for the relevant engine personnel.

\section{References}

[1] Li Bin and other editor. Main propulsion power device [M]. Dalian: Dalian Maritime University Press, Beijing: People's Communications Press, 2012.08

[2] Wang Chunfang, Ye Weijiang editor. Marine automation [M]. Dalian: Dalian Maritime University Press, 2011.02

[3] Mann Enterprise (MAN). Main propulsion device completion information. 